\title{
Designing vaccines for the immunocompromised
}

A new vaccination strategy could offer additional protection for individuals whose compromised immune systems make them a prime target for infection. The millions of patients with severely reduced concentrations of $\mathrm{CD}^{+}{ }^{+} \mathrm{T}$ cells-due to such causes as HIV infection-live in constant fear of opportunistic infections; moreover, pathogens such as the pneumonia-causing fungus Pneumocystis carinii (PC) pose a far greater threat to these individuals than to persons with healthy immune systems. Conventional vaccine strategies become ineffective, because immunocompromised patients are unable to mount an adequate protective immune response against therapeutic antigens.

One possible solution involves the use of CD40 ligand (CD40L), a peptide that triggers dendritic cell (DC) activation, followed by helper T cell-independent activation of B lymphocytes and cytotoxic T lymphocytes (CTLs). A few years ago, Jay Kolls and colleagues at the Louisiana State University Medical Center produced immunodeficient mice with DCs that express CD40L. After injecting these mice with PC antigens, his team was able to raise a relatively effective humoral immune response. More recently, Kolls — now at the University of Pittsburgh School of Medicine-and his group have expanded on this work, and in a new article from the Journal of Clinical Investigation (23 November) they present enhancements that could provide a practical vaccination strategy for use with immnocompromised patients.

Kolls' team turned to a 'prime-boost' DNA vaccination strategy, in which mice were 'primed' first with plasmid DNA containing the genes for CD40L and for the PC antigen OVA, followed weeks later by a 'boost' vaccination, with the same genes expressed from an adenoviral construct. This approach considerably enhanced the immune response of mice that had been chemically depleted of helper T cells, with production of antigenspecific antibodies and CTLs that matched amounts seen in vaccinated, immunocompetent mice. They obtained similar results in subsequent DNA vaccination experiments in genetically CD4-deficient mice using a different PC antigen, Kexin. The Kexin DNA vaccinations raised antisera that efficiently killed PC pathogens in vitro, and

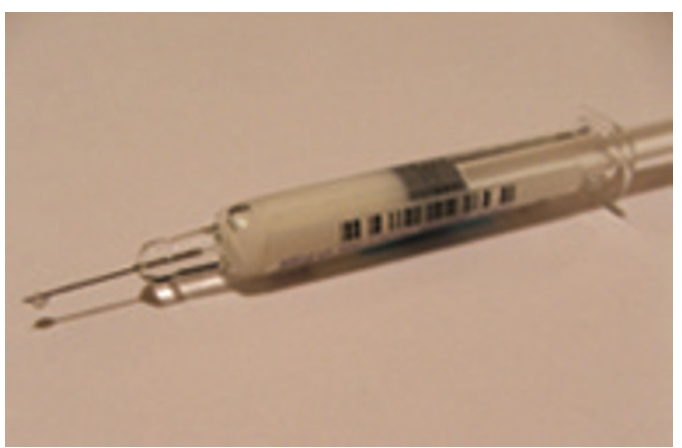

treated mice showed considerably improved protection against infection after inoculation with PC organisms. For both Kexin and OVA, coexpression of CD40L was essential for immune stimulation.

This CD40L-based DNA vaccination strategy could offer a practical clinical solution for some patients, and the authors conclude that "although the safety and efficacy of this novel vaccine approach will need to be validated in other systems, the data presented here show promise in eliciting strong cellular and humoral responses in the absence of CD4+ T cells."

Michael Eisenstein

\section{PET PROJECT REVEALS TMMUNE RESPONSE TO CANCER}

Using positron emission tomography (PET) imaging, a group of researchers at the University of California, Los Angeles, has developed a method to observe, in real time, a mouse's immune response to cancer. This technique may allow faster testing of cancer immunotherapies, as well as a way to measure the effectiveness of treatments in individual patients.

Immunotherapies represent a promising approach to treating malignancies. Indeed some of these therapies, such as the use of monoclonal antibodies, are now in general use for the treatment of some cancers. However, the lack of tools for monitoring the effectiveness of immunotherapies is limiting the speed with which they can be developed and tested. Current techniques are invasive and cannot provide a whole-body view of the immune response; now, though, a group led by Owen $\mathrm{N}$. Witte has used a noninvasive imaging technique to observe the primary immune response of chimeric mice to a cancer challenge.

PET scanners work by detecting molecules labeled with positron-emitting radioisotopes as they accumulate in metabolically active tissues. PET imaging allows for the in vivo observation and quantification of metabolic processes.

Witte's team injected hematopoietic cells that had been transduced with a PET reporter gene (sr39tk) into mice that had been lethally irradiated to eliminate their immune system. After allowing the transduced cells to engraft and re-establish an immune system, the researchers challenged the mice with a retrovirus that induces a highly immunogenic rhabdomyosarcoma. After injecting the mice with $\left[{ }^{18} \mathrm{~F}\right] \mathrm{FHBG}$, a PET substrate that accumulates in cells when phosphorylated by the sr39tk gene product, the research group used PET imaging to locate and quantify the transduced immune cells as they responded to the tumor (Proc. Natl. Acad. Sci. USA, 29 November 2005).

In addition, by injecting the mice with the radiolabeled glucose analog $\left[{ }^{18} \mathrm{~F}\right] \mathrm{FDG}$, which accumulates in highly metabolically active cells, Witte's group was able to use PET imaging to visualize the location and any changes in size of the tumor mass.

Witte tells Lab Animal, "Our next steps will be to use cell typespecific promoters to define the different types of immune cells ( $T, B$, macrophage, etc.) that participate in the inflammatory and programmed immune response to the tumor over time."

He adds, "Clinical application of the $\left[{ }^{18} \mathrm{~F}\right] \mathrm{FDG}$ PET for immune reactivity could come very soon, since this is an established clinical approach used mainly in cancer and [central nervous system] evaluation."

Tanja Schub 